\title{
Extracellular proteolytic enzymes produced by human pathogenic Vibrio species
}

\author{
Shin-ichi Miyoshi* \\ Graduate School of Medicine, Dentistry and Pharmaceutical Sciences, Okayama University, Okayama, Japan
}

\section{Edited by: \\ Daniela Ceccarelli, University of \\ Maryland, USA}

Reviewed by:

Li Sun, Institute of Oceanology, Chinese Academy of Sciences, China

Jung Sup Lee, Chosun University,

South Korea

*Correspondence:

Shin-ichi Miyoshi, Graduate School of Medicine, Dentistry and

Pharmaceutical Sciences, Okayama University, 1-1-1, Tsushima-Naka,

Kita-Ku, Okayama 700-8530, Japan e-mail:miyos-s@cc.okayama-u.ac.jp

\begin{abstract}
Bacteria in the genus Vibrio produce extracellular proteolytic enzymes to obtain nutrients via digestion of various protein substrates. However, the enzymes secreted by human pathogenic species have been documented to modulate the bacterial virulence. Several species including Vibrio cholerae and $V$. vulnificus are known to produce thermolysinlike metalloproteases termed vibriolysin. The vibriolysin from $V$. vulnificus, a causative agent of serious systemic infection, is a major toxic factor eliciting the secondary skin damage characterized by formation of the hemorrhagic brae. The vibriolysin from intestinal pathogens may play indirect roles in pathogenicity because it can activate protein toxins and hemagglutinin by the limited proteolysis and can affect the bacterial attachment to or detachment from the intestinal surface by degradation of the mucus layer. Two species causing wound infections, $V$. alginolyticus and $V$. parahaemolyticus, produce another metalloproteases so-called collagenases. Although the detailed pathological roles have not been studied, the collagenase is potent to accelerate the bacterial dissemination through digestion of the protein components of the extracellular matrix. Some species produce cymotrypsin-like serine proteases, which may also affect the bacterial virulence potential. The intestinal pathogens produce sufficient amounts of the metalloprotease at the small intestinal temperature; however, the metalloprotease production by extraintestinal pathogens is much higher around the body surface temperature. On the other hand, the serine protease is expressed only in the absence of the metalloprotease.
\end{abstract}

Keywords: Vibrio, vibriolysin, thermolysin, collagenase, serine protease

\section{HUMAN PATHOGENIC VIBRIO SPECIES}

Bacteria in the genus Vibrio are normal habitants of aquatic environments and play important roles in maintaining the aquatic ecosystem. Although more than 100 species are currently in this genus, at least, 11 species listed in Table $\mathbf{1}$ are human pathogens (Janda et al., 1988; Chakraborty et al., 1997). Amongst, Vibrio hollisae is recommended to place into a novel genus Grimontia (Thompson et al., 2003). Human pathogenic species can be classified into two groups according to types of infectious diseases: the group causing gastrointestinal diseases and that causing extraintestinal diseases. Representative species in the former group are $V$. cholerae and V. parahaemolyticus, whereas $V$. vulnificus is the most important species in the latter group. Two species, V. damsela and $V$. vulnificus, are also fish pathogens.

Of human pathogenic vibrios, $V$. cholerae is the most extensively studied species because it is a causative agent of severe watery diarrheal disease, cholera. Cholera is characterized by uncontrolled purging of copious rice water stools leading to serious electrolyte depletion, dehydration, acidosis, shock, and, if left untreated, to death. Although $V$. cholerae is divided serologically into more than 200 groups, only two serogroups, O1 and O139, are etiologic agents of epidemic cholera. The non-O1/non-O139 serogroups are etiologic agents causing sporadic diarrheal cases and occasionally extra-intestinal infections, including skin, ear, sputum, urine, and cerebrospinal fluid infections. Cholera toxin (CT) produced by the $\mathrm{O} 1$ and $\mathrm{O} 139$ serogroups is a major toxic factor evoking severe watery diarrhea, whereas, hemolysin (Shinoda and Miyoshi, 2006), Zonula occludens toxin (Fasano et al., 1991), and accessory CT (Trucksis et al., 1993) have also been demonstrated to be additional enterotoxic factors.

Vibrio parahaemolyticus inhabits commonly coastal and estuary areas in the tropical and temperate regions, but this species has been recognized to cause gastroenteritis following consumption of seafood. The outstanding features of gastroenteritis are severe abdominal pain, diarrhea (frequently bloody stools), nausea, vomiting, mild fever, and headache. The mean incubation period is 6 to $12 \mathrm{~h}$ and diarrhea or soft stools persist for 4-7 days. Although the food poisoning caused by $V$. parahaemolyticus had been frequent in Japan, the number of outbreaks has been decreased drastically in the last 10 years. Production of the hemolysin designated as thermostable direct hemolysin is closely related to the bacterial pathogenicity (Shinoda and Miyoshi, 2006). V. parahaemolyticus also causes wound-infection through exposure of a new wound to contaminated seawater or estuarine water, whereas this type of diseases is independent of production of the hemolysin.

The first clinical isolation of $V$. vulnificus was from a human leg ulcer (Roland, 1970). However, because of similar bacteriological characteristics of the bacterium isolated, this case was reported as an extra-intestinal infectious disease by $V$. parahaemolyticus (Hollis et al., 1976). V. vulnificus causes two types of infections, the primary septicemia and the wound-infection, 
Table 1 | Infectious diseases caused by Vibrio species.

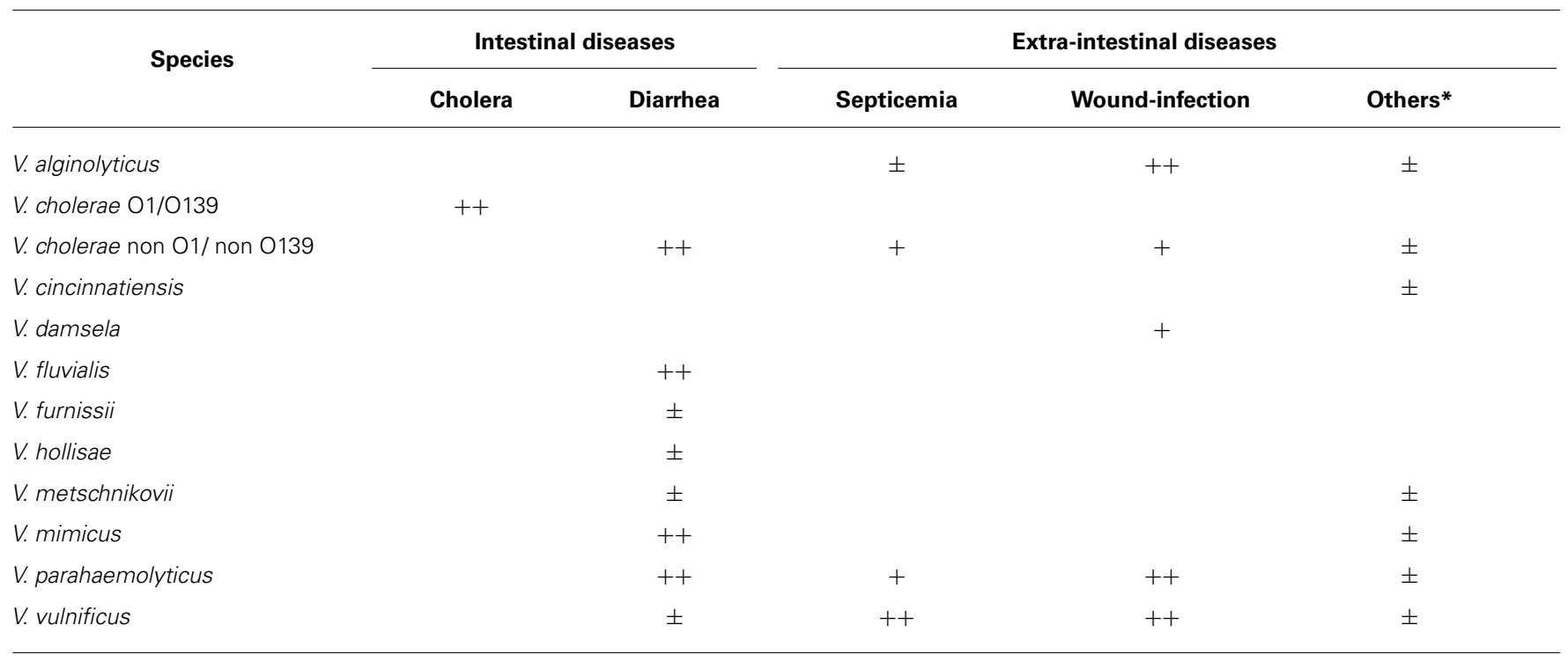

++, Major disease; +, Minor disease; \pm , Rare disease.

*Include otitis media, cholesystitis, meningitis

to human (Blake et al., 1980; Klontz et al., 1988). The primary septicemia is associated with consumption of contaminated raw seafood, especially shellfish such as oysters. However, this type is a typical opportunistic infection. Namely, most patients have an underlying disease(s) of liver dysfunction, alcoholic cirrhosis or hemochromatosis, which leads to increase in the plasma ferric ion level and to decrease in the activity of the innate immunity (Strom and Paranjpye, 2000; Inoue et al., 2008). In two-thirds of the patients, the edematous and/or hemorrhagic secondary skin lesions appear on the extremities and the trunk (Blake etal., 1980; Inoue et al., 2008). The wound-infection is characterized by the development of edema, erythema or necrosis around a new wound exposed to seawater contaminated with V. vulnificus (Blake et al., 1980; Klontz et al., 1988). This type of infection can occur in healthy persons, as well as in the immuno-compromised hosts, and may occasionally progress to septicemia.

\section{BACTERIAL EXTRACELLULAR PROTEOLYTIC ENZYMES}

Human pathogenic vibrios produce various extracellular factors including enterotoxin, hemolysin, cytotoxin, protease, collagenase, phospholipase, siderophore, and hemagglutinin (Janda et al., 1988). Of these factors, enterotoxin, hemolysin, and cytotoxin are directly related to the clinical symptoms; however, siderophore and hemagglutinin may play roles in the establishment of the infection.

Proteolytic enzymes hydrolyzing a peptide bond in proteins and peptides are essential for the homeostatic control in both eukaryotes and prokaryotes, and thus, the bacterial enzymes also have various physiological roles in the life cycle of the microorganisms. However, the enzymes produced by pathogenic species occasionally act as toxic factors to the infected host (Hase and Finkelstein, 1993; Harrington, 1996). Proteolytic enzymes are classified into several groups, such as aspartic, cysteine, and serine protease, and metalloprotease; however, many of the bacterial toxic proteases are in the metalloprotease group, which often contains a zinc (II) ion in the catalytic center (Hase and Finkelstein, 1993; Hooper, 1994). As shown in Table 2, human pathogenic Vibrio species also produce and secrete proteolytic enzymes, and several enzymes have been extensively characterized as direct toxic factors causing skin damage or indirect virulence factors processing other protein toxins. The enzymes produced by vibrios are in two metalloprotease groups (vibriolysin and collagenases) or one serine protease group (chymotrypsin-like proteases).

The progress in the molecular biology has provided much information on the DNA-derived amino acid sequences of metalloproteases and has shown the presence of the consensus sequence HEXXH as the zinc-binding motif. This motif was also found in some bacterial protein toxins including clostridial neurotoxins,

Table 2 | Extracellular proteolytic enzymes produced by pathogenic Vibrio species.

\begin{tabular}{|c|c|c|c|}
\hline \multirow{2}{*}{ Species } & \multicolumn{2}{|c|}{ Metalloprotease } & \multirow{2}{*}{$\begin{array}{c}\text { Serine protease } \\
\begin{array}{c}\text { Chymotrypsin-like } \\
\text { protease }\end{array}\end{array}$} \\
\hline & Vibriolysin & Collagenase & \\
\hline V. alginolyticus & & + & + \\
\hline V. cholerae & + & & \\
\hline V. fluvialis & + & & \\
\hline V. metschnikovii & & & + \\
\hline V. mimicus & + & & \\
\hline V. parahaemolyticus & & + & + \\
\hline V. vulnificus & + & & + \\
\hline
\end{tabular}


Bacteroides fragilis enterotoxin, and Bacillus anthracis lethal factor. Indeed, these bacterial toxins were verified to show the remarkably specific proteolytic action toward a target host protein (Miyoshi and Shinoda, 2000). For instance, clostridial neurotoxins can cleave the restricted protein components of the neuroexocytosis machinery, which leads to the blockade of neurotransmitter release and consequent muscle paralysis (Schiavo et al., 1993). In addition, a novel cytotoxin that consists of one A subunit and five B subunits was isolated from some enterohemorrhagic Escherichia coli strains, and the A subunit was indicated to be a subtilase-like serine protease (Paton and Paton, 2010). The RTX (repeated-intoxin) toxins are large multifunctional cytotoxins and are possible to modulate the virulence of a number of gram-negative bacterial pathogens including $V$. cholerae and V. vulnificus (Satchell, 2007; Prochazkova et al., 2009; Roig et al., 2011). In V. cholerae RTX toxin, the cysteine protease domain was reported to mediate autoprocessing of the toxin (Sheahan et al., 2007; Shen et al., 2009).

\section{VIBRIOLYSIN \\ BIOCHEMICAL PROPERTIES}

Zinc-containing metalloproteaes consist of four superfamilies based on the amino acid residues in the zinc-binding site, and the zincins superfamily is characterized by the possessing of the HEXXH motif (Hooper, 1994). The thermolysin family, in which prototype enzyme is thermolysin from Bacillus thermoproteolyticus, is a one of major members of this superfamily. The enzymes in this family are commonly characterized by the presence of Glu at the 25th position from the first His of the above motif. $V$. proteolyticus is a marine microorganism that was first isolated from the intestine of a small, wood-boring isopod crustacean (Merkel et al., 1964). Griffin and Prescott (1970) purified a highly active metalloprotease from the bacterial culture supernatant. Durham (1989) first disclosed the designation of this enzyme as vibriolysin in the patent literature. Thereafter, highly homologous metalloproteases have been isolated from other Vibrio species including human pathogens V. cholerae, $V$. fluvialis, V. mimicus, and V. vulnificus. Therefore, the name of vibriolysin is currently applicable to all of these proteolytic enzymes (Miyoshi et al., 2012a).

Vibriolysin hydrolyzes specifically the peptide bond at the amino group side of the $\mathrm{P}^{\prime}$ amino acid residue, which is usually a hydrophobic amino acid reside (e.g., Phe, Tyr, or Leu; Narukawa et al., 1993). Synthetic oligopeptides, such as carbobenzoxy (Z)-Gly-Phe- $\mathrm{NH}_{2}$ and Z-Gly-Leu- $\mathrm{NH}_{2}$, are thus commonly used as the suitable substrate. On the other hand, phosphoramidon [N-( $\alpha$-rhamnopyranosyloxy-hydroxyphosphanyl)Leu-Trp] and zincov [2-( $N$-hydroxycarboxamido)-4-methylpentanoyl-AlaGly amide] are well-known competitive inhibitors. In addition, phenylazobenzyloxycarbonyl-Pro-Leu-Gly-Pro-Arg, the substrate developed for bacterial collagenases, is significantly hydrolyzed by the enzyme (Miyoshi etal., 1987b). Vibriolysin is also highly active on a wide variety of protein substrates. Namely, the enzyme exhibits significant proteolysis of casein, albumin, hemoglobin, type I and IV collagen, gelatin, elastin, fibrin, and fibrinogen (Miyoshi et al., 1995; Miyoshi and Shinoda, 2000).

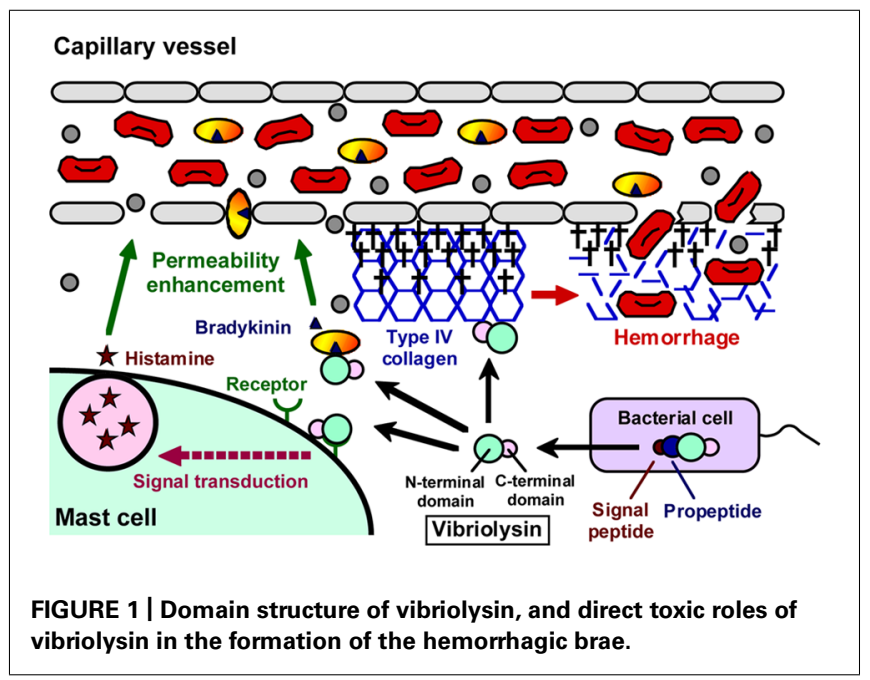

Like thermolysin, vibriolysin is synthesized as an inactive precursor, and maturation of the precursor is achieved by several processing stages (Chang et al., 2007). In the case of the enzyme from V. vulnificus (Figure 1), it is initially synthesized as the preproenzyme (609 aa, 65,964 Da) with a typical signal peptide (Cheng et al., 1996). The signal peptide is cleaved during its passage through the inner membrane in the signal peptidase-dependent manner. In the periplasm, the propeptide that may function as an intramolecular chaperone mediating maturation of the enzyme and/or a specific inhibitor to protect autodigestion of the enzyme (Chang et al., 2007) is then cleaved by an autocatalytic mechanism, and finally, the mature vibriolysin (413 aa, 44,648 Da) is generated. The vibriolysin maturated consists of two functional domains: the N-terminal domain (314 aa, 34,049 Da) mediating the catalytic action, and the C-terminal domain (99 aa, 10,656 Da) essential for efficient attachment to protein substrates (Yun et al., 2012). The $\mathrm{N}$-terminal domain is easily obtained by autocatalytic limiteddigestion of the C-terminal domain (Miyoshi et al., 1997). The $\mathrm{N}$-terminal domain alone possesses sufficient proteolytic activity toward oligopeptides or soluble proteins, while it shows markedly reduced activity toward insoluble proteins such as type I collagen and elastin.

The N-terminal domain of vibriolysin is significantly related to other enzymes in the thermolysin family; however, its C-terminal domain may be unique. The feature of the proteolytic action, which is mediated by the N-terminal domain, is considerably similar to other enzymes. However, neither suitable specific peptide substrate nor competitive inhibitor for vibriolysin has been developed. On the other hand, the hemagglutinating action on rabbit erythrocytes, which is due to association of both $\mathrm{N}$-terminal and C-terminal domains with the erythrocyte membrane, is a distinctive feature of vibriolysin so far as reported (Alam et al., 1995).

\section{PATHOLOGICAL ROLES}

As summarized in Figure 2, a variety of pathological roles of vibriolysin have been documented (Shinoda and Miyoshi, 2011). In the local infections such as the wound-infection, vibriolysin is thought to be a direct toxic factor that causes hemorrhagic tissue 


\section{Wound- \\ infection}

Direct toxic factor

1) Hemorrhagic tissue damage

Destruction of the basement membrane by digestion of type IV collagen

2) Enhancement of the vascular permeability and edema

Exocytotic histamine release from mast cells

Generation of bradykinin through activation of the cascade

3) Acceleration of the bacterial dissemination

Digestion of components of the extracellular matrix

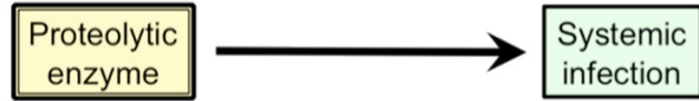

Virulence modulator Synergistic pathogenic factor

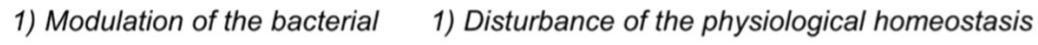

attachment or detachment Confusion of the protease-protease inhibitor systems

2) Activation of protein toxins Acceleration of the heme utilization

Intestinal

infection

FIGURE 2 | Pathological roles of extracellular proteolytic enzymes in infectious diseases caused by Vibrio species.

damage through digestion of the basement membrane around vascular endothelial cells, and that forms edematous lesions through generation of inflammatory mediators (Figure 1). The enzyme from $V$. vulnificus can enhance the vascular permeability when injected into the mammalian dorsal skin. In rat skin, this reaction is due to the release of histamine from mast cells, because the vascular enhancement was abolished by simultaneous injection of diphenhydramine, an anti-histaminic agent (Miyoshi et al., 1987c). In guinea pig skin, however, the permeability enhancement is most likely due to activation of the factor XII-plasma kallikrein-kinin cascade (Miyoshi et al., 1987a). Namely, the skin reaction was not blocked by diphenhydramine, but it was modulated by in situ administration of the specific inhibitors affecting the cascade activation. Bradykinin, a well-known in vivo mediator of inflammation, is finally generated through activation of the cascade. Further studies to clarify the activation mechanism of the human cascade have been carried out, and the results demonstrated that vibriolysin could generate the active forms via limited proteolysis of the inactive zymogens (Miyoshi et al., 2004). Plasma prekallikrein was converted to the active kallikrein, which can liberate bradykinin from kininogen, by cleavage of the $\mathrm{Arg}^{371}-\mathrm{Ile}^{372}$ bond. On the other hand, factor XII was activated by hydrolysis of the $\mathrm{Arg}^{353}-\mathrm{Val}^{354}$ or Gly ${ }^{357}$-Leu ${ }^{358}$ bond, and activated factor XII could convert plasma prekallikrein to kallikrein. Vibriolysin also induces the hemorrhagic reaction in the mammalian dorsal skin. The enzyme from V. vulnificus showed the greatest hemorrhagic activity compared with some bacterial metalloproteases, thermolysin from $B$. thermoproteolyticus, serralysin from Serratia species, and collagenase from Clostridium histolyticum (Miyoshi et al., 1998). The levels of the in vivo hemorrhagic activities of these proteases were correlated with those of the in vitro proteolytic activities toward the reconstituted basement membrane gel. Of two major basement components, laminin and type IV collagen, only the latter was easily digested by vibriolysin. This indicates that type IV collagen forming the framework of the basement membrane is the target protein. Therefore, specific degradation of type IV collagen causes destruction of the basement membrane, breakdown of capillary vessels, and finally the leakage of blood components including erythrocytes.

In the systemic infections including septicemia, vibriolysin may act as a synergistic pathogenic factor through disordered proteolysis of various plasma proteins, which in turn disturbs the physiological homeostasis, and eventually elicits an immunocompromised state in the infected host. For instances, vibriolysin has been documented to facilitate the bacterial infection by disturbance of the plasma protease-protease inhibitor systems (Miyoshi et al., 1995), and to interfere with the blood homeostasis through prothrombin activation and fibrinolysis (Chang etal., 2005; Kwon etal., 2007). Acceleration of the heme utilization was also reported as the pathogenic role of vibriolysin (Nishina etal., 1992). Ferric/ferrous ion is essential for in vivo growth of pathogenic microorganisms. However, the concentration of free ferric/ferrous ion in human body is very low $\left(10^{-15}\right.$ to $10^{-18} \mathrm{M}$ ) because of the presence of heme-proteins including hemoglobin and iron-binding proteins. Therefore, pathogenic microorganisms invaded into human body must operate the systems to acquire ferric/ferrous ion. Heme, a complex of porphyrin with ferric/ferrous ion and a prosthetic group in hemoglobin or other heme-proteins, is often used as an iron source by human pathogenic bacterial species (Stojiljkovic and Perkins-Balding, 
2002; Tong and Guo, 2009). Nishina et al. (1992) documented that the vibriolysin-deficient mutants of $V$. vulnificus could not grow in the iron-restricted broth because of the inability to utilize hemoglobin as an iron source, but the bacterial growth was apparently restored by the addition of the purified vibriolysin. On the other hand, hemoglobin or ferric ion is known to be required for efficient transcription of the gene encoding the vibriolysin (Kawase et al., 2004; Sun et al., 2006). Incidentally, it should be noted that human plasma contains a broad-range protease inhibitor $\alpha_{2}$-macroglobulin $\left(\alpha_{2} M\right)$ as a primary inhibitor for exogenous proteolytic enzymes including bacterial enzymes. Vibriolysin was also inactivated with $\alpha_{2} \mathrm{M}$ at a molar ratio of 1:1 by means of physical entrapment (Miyoshi and Shinoda, 1989). Therefore, the pathological actions of vibriolysin documented herein are possible to support the systemic infection but may be highly restricted in vivo.

Vibriolysin from enteric pathogens may increase the bacterial attachment to the intestinal surface through digestion of the mucosal substances or the bacterial outer membrane proteins (Alam et al., 2006). However, the inverse effect of the enzyme was also known. The vibriolysin from $V$. cholerae $\mathrm{O} 1$ was reported to accelerate the bacterial detachment from cultured cells by digestion of V. cholerae adhesins (Finkelstein et al., 1992). Vibriolysin also modulate the enterotoxicity of the bacterial toxins by limited proteolysis. For instance, the enzyme from $V$. cholerae $\mathrm{O} 1$ can activate CT through nicking of the A subunit of CT (Booth et al., 1984). Vibriolysin also converts the precursor of the enterotoxic hemolysin produced by $V$. cholerae to the mature toxin through removal of the $15 \mathrm{kDa}$-terminal propeptide (Nagamune et al., 1996). Although the results shown above suggest indirect pathogenic roles of vibriolysin, the possibility of the direct roles has also been reported. Ghosh et al. (2006) purified vibriolysin from a CT gene-negative strain of $V$. cholerae non-O1/non-O139 and measured the enterotoxic activity. The purified enzyme caused accumulation of the hemorrhagic fluid in the rabbit ileal loop assay and increase in the intestinal short-circuit current in the Using chamber assay. Additionally, through the analysis with several mutants genetically constructed, Silva et al. (2006) showed that vibriolysin was necessary for full expression of enterotoxicity of $V$. cholerae $\mathrm{O} 1$.

\section{COLLAGENASES}

Yu and Lee (1999) and Kim et al. (2002) carried out cloning of the $V$. parahaemolyticus gene encoding a collagenase, which was designated as PrtV/PrtVp (562 aa, 63,156 Da) and VppC (814 aa, $89,833 \mathrm{Da}$ ), respectively. These enzymes were revealed to be metalloproteases in the zincins superfamily having the consensus zinc-binding HEXXH motif, but neither of the collagenases was in the thermolysin family. Miyoshi et al. (2008) showed that, only when $V$. parahaemolyticus was cultivated at $26^{\circ} \mathrm{C}$, the $v p p C$ gene was sufficiently expressed, and $\mathrm{VppC}$ was secreted from the bacterial cell after removal of the N-terminal 72 amino acid residues. In contrast, expression of the prtV/prtVp gene was negligible in the wild type strain. The gene was significantly expressed by disruption of the $v p p C$ gene; however, the product PrtV/PrtVp was not secreted into the cultivation broth (Miyoshi et al., 2008), suggesting PrtV/PrtVp is a cell-associated enzyme. VppC purified showed the steady activity to hydrolyze Z-Gly-Pro-Gly-Gly-ProAla, the specific substrate for bacterial collagenases, and to digest gelatin. This indicates that $\mathrm{VppC}$ may contribute to the woundinfection by $V$. parahaemolyticus because putative digestion of the components of the extracellular matrix by VppC may accelerate the bacterial dissemination and may form cellulitic skin damage. $V$. alginolyticus, another species causing the woundinfection, is known to produce a VppC homolog (Takeuchi et al., 1992).

\section{CHYMOTRYPSIN-LIKE PROTEASES}

In 2002, two research groups reported individually purification of a serine protease, which was termed protease A and VPP1 respectively, from the culture supernatant of $V$. parahaemolyticus (Ishihara et al., 2002; Lee et al., 2002). These proteases were identical and corresponded to the VPA0227 protein (677 aa, $71,038 \mathrm{Da}$ ) of strain RIMD 2210633 (GenBank accession number: BA000032). However, the amino acid sequencing of the purified enzyme indicated that the $\mathrm{N}$-terminal 121 amino acid residues were removed during the maturating process. This serine protease designated herein as protease A/VPP1 showed the immunological cross-reactivity with serine proteases from $V$. metschnikovii and $V$. alginolyticus (Ishihara et al., 2002). Indeed, protease A/VPP1 revealed highly similarity of the amino acid sequence to the enzyme from V. metschnikovii (Kwon et al., 1995). Production of protease A/VPP1 was much higher at 25 than $37^{\circ} \mathrm{C}$ and was induced by the addition of gelatin to the cultivation broth. Miyoshi et al. (2008) reported that production of this serine protease was remarkably increased by disruption of the $v p p C$ gene, suggesting that protease A/VPP1 is a substitutive enzyme of VppC. The purified protease was found to be sensitive to chymostatin, the well-known competitive inhibitor for chymotrypsin, and to hydrolyze the specific peptidyl-4-methyl-coumaryl-7-amide (MCA) substrates for chymotripsin, such as Glutaryl-Ala-AlaPhe-MCA and Succinyl-Ala-Ala-Pro-Phe-MCA. Therefore, it may be concluded that protease A/VPP1 is a chymotrypsin-like serine protease. The cytotoxicity against $\mathrm{CHO}, \mathrm{HeLa}$, or Vero cells and the mouse lethal toxicity of purified protease A/VPP1 was demonstrated by Lee et al. (2002). Our preliminary study showed the proteolytic activity of the purified enzyme toward extracellular matrix components, laminin and type I collagen. These results suggest that protease A/VPP1 also modulate the bacterial pathogenicity.

Vibrio vulnificus sometime causes severe hemorrhagic septicemia called vibriosis in eels of the culture farms (Tison et al., 1982; Biosca et al., 1991). A few strains isolated from diseases eels were recently found to have lost the $80 \mathrm{~kb}$ genomic region including the gene encoding vibriolysin, but instead of vibriolysin, these strains secrete a serine protease termed VvsA, which is an ortholog of protease A/VPP1 from $V$. parahaemolyticus (Miyoshi et al., 2012b). As protease A/VPP1, production of VvsA was extremely increased in the absence of the functional gene encoding vibriolysin (Wang et al., 2008). The vvsA gene constitutes an operon with a downstream gene $v v s B$, of which product $\mathrm{VvsB}$ may act as the chaperon supporting the maturation process of VvsA. The database analysis showed that several Vbrio species including $V$. parahaemolyticus have the homologus genes to $v v s A B$, indicating 
widely distribution of the chymotrypsin-like serine protease in Vibrio species.

\section{REGULATION OF PRODUCTION OF PROTEOLYTIC ENZYMES}

Vibrio species are ubiquitous microorganisms in aquatic environments, but 11 species cause intestinal or extra-intestinal infections to humans. During the infection process, the bacterial cells must sense the change of environmental factors, such as temperature, $\mathrm{pH}$, salinity, and osmolarity, and then, the bacterium must transmit the signals into the cells through the specific signaltransduction systems, which result in the change of expression of the genes. Especially, the genes encoding the toxic or virulence factors, which may be required for in vivo survival and growth, must be expressed at an appropriate place and time in a tightly regulated fashion (Heithoff et al., 1997; Lee et al., 1999). Amongst the environmental factors, temperature is thought to be the most important. The vibriolysin genes of the bacterium causing intestinal infections are expressed sufficiently at $37^{\circ} \mathrm{C}$, while the genes of the bacterium causing extra-intestinal infections are expressed more effectively around the body surface temperature (Watanabe et al., 2004; Miyoshi et al., 2006). The gene expression is also often affected by the salinity. For instance, the expression level of the $\operatorname{pppC}$ gene in $V$. parahaemolyticus is higher at $3 \% \mathrm{NaCl}$ than $0.9 \% \mathrm{NaCl}$ (Miyoshi et al., 2008). Although the intracellular second messengers, such as cyclic AMP and cyclic di-GMP, and the global general regulators including RpoS and histone-like nucleotide structuring protein are also involved in the gene regulation (Benitez et al., 2001; Wang et al., 2011, 2012), the molecular mechanisms how the bacterium senses the environmental signals and transmits the signals into the cells are not clarified.

Production of proteolytic enzymes is tightly dependent on the growth phase and reaches to the maximum level at the early stationary phase. Many pathogenic bacteria coordinate expression of the virulence genes in response to the bacterial cell density. This regulation system is termed the quorum sensing (QS) and is controlled by the small diffusible signal molecule called autoinducer (AI; Whitehead et al., 2001; Henke and Bassler, 2004). At the low cell density, the QS system cannot modulate the gene expression because the concentration of AI is too low. However, at the high cell density, the AI concentration around the bacterial cell reaches the threshold level, the AI molecule is sensed by the sensor protein, the signals are transmitted into the cell, and finally, the expression of a set of genes is started or stopped.

In Vibrio species including V. cholerae, V. mimicus, and V. vulnificus (Miller et al., 2002; Milton, 2006; Sultan et al., 2006), the AI molecule is detected by the specific membrane-bound sensor protein, which causes conversion of the sensor protein from kinase to phosphatase. Subsequently, the sensor protein/phosphatase mediates dephosphorylation of LuxU-LuxO, the response regulator proteins. The dephosphorylated LuxO has no activity to inhibit LuxR or its homolog, the master transcriptional regulator for the genes under the control of the QS system. Therefore, at the high cell density, the transcriptional status of the target genes is changed by the function of LuxR or its homologue. Production of the proteolytic enzymes by pathogenic vibrios is closely related with the extracellular AI level (Kim et al., 2003; Kawase et al., 2004;
Raychaudhuri etal., 2006). For instance, the mutant of the AI synthetase showed apparently reduced production of vibriolysin. Therefore, the QS system markedly controls the expression of the proteolytic genes in Vibrio species. However, it should be noted that the QS system of $V$. cholerae or V. mimicus is operated sufficiently at $37^{\circ} \mathrm{C}$ (Sultan et al., 2006), whereas, the system of $V$. vulnificus is operated effectively at $26^{\circ} \mathrm{C}$ but not at $37^{\circ} \mathrm{C}$ (Miyoshi et al., 2006).

\section{CONCLUSION}

The proteolytic enzymes produced by human pathogenic Vibrio species may play a variety of pathological roles: direct roles by digesting many kinds of host proteins or indirect roles by processing other toxic protein factors. Especially, vibriolysin from V. vulnificus is thought to be a major virulence factor. However, some contradictions of the pathogenic roles were also reported (Shao and Hor, 2000; Sun et al., 2006). It must be mentioned that the purified enzymes from $V$. vulnificus and $V$. proteolyticus, a non-pathogenic species, are difficult to distinguish in the in vivo actions, because both enzymes are members of vibriolysin and have comparative biochemical and toxic activities. However, it has been demonstrated that the high growing ability of $V$. vulnificus in the mammal host is important for the pathogenicity of the bacterium (Watanabe et al., 2004). In addition, production of the toxic or virulence factors including proteolytic enzymes is tightly regulated by environmental factors, the bacterial cell density and so on. Therefore, the overall experiments from various approaches are necessary for evaluation of the extracellular proteolytic enzymes as the virulence factors.

\section{REFERENCES}

Alam, M., Miyoshi, S., Ahmed, K. U., Hasan, N. A., Tomochika, K., and Shinoda, S. (2006). Proteolytic activation of Vibrio mimicus (Vm) major outer membrane protein haemagglutinin (HA) with Vm-HA/protease: implication for understanding bacterial adherence. Microbiol. Immunol. 50, 845-850.

Alam, M., Miyoshi, S., and Shinoda, S. (1995). Production of antigenically related exocellular elastolytic proteases mediating hemagglutination by vibrios. Microbiol. Immunol. 39, 67-70.

Benitez, J. A., Silva, A. J., and Finkelstein, R. A. (2001). Environmental signals controlling of hemagglutinin/protease in Vibrio cholerae. Infect. Immun. 69, 65496553. doi: 10.1128/IAI.69.10.6549-6553.2001

Biosca, E. G., Amaro, C., Esteve, C., Alcaide, E., and Garay, E. (1991). First record of Vibrio vulnificus biotype 2 from diseased European eel, Anguilla anguilla. J. Fish Dis. 14, 103-109. doi: 10.1111/j.1365-2761.1991.tb00581.x

Blake, P. A., Weaver, R. E., and Hollis, D. G. (1980). Disease of humans other than cholera) caused by vibrios. Annu. Rev. Microbiol. 34, 341-367. doi: 10.1146/annurev.mi.34.100180.002013

Booth, B. A., Boesman-Finkelstein, M., and Finkelstein, R. A. (1984). Vibrio cholerae hemagglutinin/protease nicks cholera enterotoxin. Infect. Immun. 45, 558-560.

Chakraborty, S., Nair, G. B., and Shinoda, S. (1997). Pathogenic vibrios in the natural aquatic environment. Rev. Environ. Health 12, 63-80. doi: 10.1515/REVEH.1997.12.2.63

Chang, A. K., Ki, H. Y., Park, J. E., Acharya, P., Park, I. S., Yoon, S. M., et al. (2005). Vibrio vulnificus secretes a broad-specificity metalloprotease capable of interfering with blood homeostasis through prothrombin activation and fibrinolysis. $J$. Bacteriol. 187, 6909-6916. doi: 10.1128/JB.187.20.6909-6916.2005

Chang, A. K., Park, J. W., Lee, E. H., and Lee, J. S. (2007). The N-terminal propeptide of Vibrio vulnificus extracellular metalloprotease is both an inhibitor of and a substrate for the enzyme. J. Bacteriol. 189, 6832-6838. doi: 10.1128/JB.00396-07

Cheng, J. C., Shao, C. P., and Hor, L. I. (1996). Cloning and nucleotide sequencing of the protease gene of Vibrio vulnificus. Gene 183, 255-257. doi: 10.1016/S03781119(96)00488-X 
Durham, D. R. (1989). Cleaning compositions containing protease produced by Vibrio and method of use. US Patent 4,865,983.

Fasano, A., Baudry, B., Pumplin, D. W., Wasserman, S. S., Tall, B. D., Kelly, J. M., et al. (1991). Vibrio cholerae produces a second enterotoxin which affects intestinal tight junctions. Proc. Natl. Acad. Sci. U.S.A. 86, 5242-5246. doi: 10.1073/pnas.88.12.5242

Finkelstein, R. A., Boesman-Finkelstein, M., Chang, Y., and Hase, C. C. (1992). Vibrio cholerae hemagglutinin/protease, colonial variation, virulence, and detachment. Infect. Immun. 60, 472-478.

Ghosh, A., Saha, D. R., Hoque, K. M., Asakuna, M., Yamasaki, S., Koley, H., et al (2006). Enterotoxigenicity of mature 45-kilodalton and processed 35-kilodaton forms hemagglutinin protease prurified from a cholera toxin gene-negative Vibrio cholerae non-O1, non-O139 strain. Infect. Immun. 74, 2937-2946. doi: 10.1128/IAI.74.5.2937-2946.2006

Griffin, T. B., and Prescott, J. M. (1970). Some physical characteristics of a proteinase from Aeromonas proteolytica. J. Biol. Chem. 245, $1348-1356$.

Harrington, D. J. (1996). Bacterial collagenases and collagen-degrading enzymes and their role in human disease. Infect. Immun. 64, 1885-1891.

Hase, C. C., and Finkelstein, R. A. (1993). Bacterial extracellular zinc-containing metalloproteases. Microbiol. Rev. 57, 823-837.

Heithoff, D. M., Conner, C. P., and Mahan, M. J. (1997). Dissecting the biology of a pathogen during infection. Trends Microbiol. 5, 509-513. doi: 10.1016/S0966842X(97)01153-0

Henke, J. M., and Bassler, B. L. (2004). Bacterial social engagements. Trends Cell Biol. 14, 648-656. doi: 10.1016/j.tcb.2004.09.012

Hollis, D. G., Weaver, R. E., Baker, C. N., and Thronsberry, C. (1976). Halophilic Vibrio species isolated from blood cultures. J. Clin. Microbiol. 3 , $425-431$.

Hooper, N. M. (1994). Families of zinc metalloproteases. FEBS Lett. 354, 1-6. doi: 10.1016/0014-5793(94)01079-X

Inoue, Y., Ono, T., Matsui, T., Miyasaka, J., Kinoshita, Y., and Ihn, H (2008). Epidemiological survey of Vibrio vulnificus infection in Japan between 1999 and 2003. J. Dermatol. 35, 129-139. doi: 10.1111/j.1346-8138.2008. 00432.x

Ishihara, M., Kawanishi, A., Watanabe, H., Tomochika, K., Miyoshi, S., and Shinoda, S. (2002). Purification of a serine protease of Vibrio parahaemolyticus and its characterization. Microbiol. Immunol. 46, 298-303.

Janda, J. M., Power, C., Bryant, R. G., and Abbott, S. L. (1988). Current perspectives on the epidemiology and pathogenesis of clinically significant Vibrio spp. Clin. Microbiol. Rev. 1, 245-267.

Kawase, T., Miyoshi, S., Sultan, Z., and Shinoda, S. (2004). Regulation system for protease production in Vibrio vulnificus. FEMS Microbiol. Lett. 240, 55-59. doi: 10.1016/j.femsle.2004.09.023

Kim, S. Y., Lee, S. E., Kim, Y. R., Kim, C. M., Ryu, P. Y., Choy, H. E., et al. (2003). Regulation of Vibrio vulnificus virulence by the LuxS quorum-sensing system. Mol. Microbiol. 8, 1647-1664. doi: 10.1046/j.1365-2958.2003.03536.x

Kim, S. K., Yang, J. Y., and Cha, J. (2002). Cloning and sequence analysis of a novel metalloprotease gene from Vibrio parahaemolyticus 04. Gene 283, 277-286. doi: 10.1016/S0378-1119(01)00882-4

Klontz, K. C., Lieb, S., Schreiber, M., Janowski, H. T., Baldy, L., and Gunn, R. A. (1988). Syndromes of Vibrio vulnificus infections: clinical and epidemiological features in Florida cases, 1981-1987. Ann. Intern. Med. 109, 318-323. doi: 10.7326/0003-4819-109-4-318

Kwon, J. Y., Chang, A. K., Park, J. E., Shing, S. Y., Yoon, S. M., and Lee, J. S. (2007). Vibrio extracellular protease with prothrombin activation and fibrinolytic activities. Int. J. Mol. Med. 19, 157-163.

Kwon, Y. T., Kim, J. O., Moon, S. Y., Yoo, Y. D., and Rho, H. M. (1995). Cloning and characterization of the gene encoding an extracellular alkaline serine protease from Vibrio metschnikovii strain RH530. Gene 152, 59-63. doi: 10.1016/03781119(94)00648-C

Lee, C. Y., Cheng, M. F., Yu, M. S., and Pan, M. J. (2002). Purification and characterization of a putative virulence factor, serine protease, from Vibrio parahaemolyticus. FEMS Microbiol. Lett. 209, 31-37. doi: 10.1111/j.15746968.2002.tb11105.x

Lee, S. H., Hava, D. L., Waldor, M. K., and Camilli, A. (1999). Regulation and temporal expression patterns of Vibrio cholerae virulence genes during infection. Cell 99, 625-634. doi: 10.1016/S0092-8674(00)81551-2
Merkel, J. R., Traganza, E. D., Mukherjee, B. B., Griffin, T. B., and Prescott, J. M. (1964). Proteolytic activity and general characteristics of a marine bacterium, Aeromonas proteolytica sp. N. J. Bacteriol. 87, 1227-1233.

Miller, M. B., Skorupski, K., Lenz, D. H., Taylor, R. K., and Bassler, B. L. (2002). Parallel quorum sensing systems converge to regulate virulence in Vibrio cholerae. Cell 110, 303-314. doi: 10.1016/S0092-8674(02)00829-2

Milton, D. L. (2006). Quorum sensing in vibrios: complexity for diversification. Int. J. Med. Microbiol. 296, 61-71. doi: 10.1016/j.ijmm.2006.01.044

Miyoshi, N., Miyoshi, S., Sugiyama, K., Suzuki, Y., Furuta, H., and Shinoda, S. (1987a). Activation of the plasma kallikrein-kinin system by Vibrio vunificus protease. Infect. Immun. 55, 1936-1939.

Miyoshi, N., Shimizu, C., Miyoshi, S., and Shinoda, S. (1987b). Purification and characterization of Vibrio vulnificus protease. Microbiol. Immunol. 31, 13-25.

Miyoshi, S., Sugiyama, K., Suzuki, Y., Furuta, H., Miyoshi, N., and Shinoda, S. (1987c). Enhancement of vascular permeability due to histamine-releasing effect of Vibrio vulnificus protease. FEMS Microbiol. Lett. 40, 95-98. doi: 10.1111/j.15746968.1987.tb01989.x

Miyoshi, S., Narukawa, H., Tomochika, K., and Shinoda, S. (1995). Actions of Vibrio vulnificus metalloprotease on human plasma proteinase-proteinase inhibitor systems: a comparative study of native protease with its derivative modified by polyethylene glycol. Microbiol. Immunol. 39, 959-966.

Miyoshi, S., Nakazawa, H., Kawata, K., Tomochika, K., Tobe, K., and Shinoda, S. (1998). Characterization of the hemorrhagic reaction caused by Vibrio vulnificus metalloprotease, a member of the thermolysin family. Infect. Immun. 66, 48514855.

Miyoshi, S., Nitanda, Y., Fujii, K., Kawahara, K., Li, T., Maehara, Y., et al. (2008). Differential expression and extracellular secretion of the collagenolytic enzymes by the pathogen Vibrio parahaemolyticus. FEMS Microbiol. Lett. 283, 176-181. doi: 10.1111/j.1574-6968.2008.01159.x

Miyoshi, S., Okamoto, K., and Takahashi, E. (2012a) "Vibriolysin," in Handbook of Proteolytic Enzymes, 3rd Ed, eds N. D. Rawlings and G. Salvesen (London: Academic Press), 579-582.

Miyoshi, S., Wang, J., Katoh, K., Senoh, M., Mizuno, T., and Maehara, Y. (2012b). An extracellular serine protease produced by Vibrio vulnificus NCIMB 2137, a metalloprotease-gene negative strain isolated from a diseased eel. World J. Microbiol. Biotechnol. 28, 1633-1639. doi: 10.1007/s11274-0110969-y

Miyoshi, S., and Shinoda, S. (1989). Inhibitory effects of 2-macriglobulin on Vibrio vulnificus proteae. J. Biochem. 106, 299-303.

Miyoshi, S., and Shinoda, S. (2000). Microbial metalloproteases and pathogenesis. Microbes Infect. 2, 91-98. doi: 10.1016/S1286-4579(00)00280-X

Miyoshi, S., Sultan, S. Z., Yasuno, Y., and Shinoda, S. (2006). Growth phasedependent production of a toxic mtalloprotease by Vibrio vulnificus. Toxin Rev. 25, 19-30. doi: $10.1080 / 15569540500320862$

Miyoshi, S., Wakae, H., Tomochika, K., and Shinoda, S. (1997). Functional domains of a zinc metalloprotease from Vibrio vulnificus. J. Bacteriol. 179, 7606-7609.

Miyoshi, S., Watanabe, H., Kawase, T., Yamada, H., and Shinoda, S. (2004). Generation of active fragments from human zymogens in the bradykinin-generating cascade by extracellular proteases from Vibrio vulnificus and V. parahaemolyticus. Toxicon 44, 887-893. doi: 10.1016/j.toxicon.2004.08.013

Nagamune, K., Yamamoto, K., Naka, A., Matsuyama, J., Miwatani, T., and Honda, T. (1996). In vitro proteolytic processing and activation of the recombinant precursor of El Tor cytolysin/hemolysin (pro-HlyA) of Vibrio cholerae by soluble hemagglutinin/protease of $V$. cholerae, trypsin, and other proteases. Infect. Immun. 64, 4655-4658.

Narukawa, H., Miyoshi, S., and Shinoda, S. (1993). Chemical modification of Vibrio vulnificus metalloprotease with activated polyethylene glycol. FEMS Microbiol. Lett. 108, 43-46. doi: 10.1111/j.1574-6968.1993.tb06071.x

Nishina, Y., Miyoshi, S., Nagase, A., and Shinoda, S. (1992). Significant role of an exocellular protease in utilization of heme by Vibrio vulnificus. Infect. Immun. 60, $2128-2132$.

Paton, A. W., and Paton, J. C. (2010). Escherichia coli subtilase cytotoxin. Toxins 2, 215-228. doi: 10.3390/toxins2020215

Prochazkova, K., Shuvalova, L. A., Minasov, G., Voburka, Z., Anderson, W. F., and Satchell, K. J. F. (2009). Structural and molecular mechanism for autoprocessing of MARTX toxin of Vibrio cholerae at multiple sites. J. Biol. Chem. 284, 2655726568. doi: 10.1074/jbc.M109.025510 
Raychaudhuri, S., Jain, V., and Dongre, M. (2006). Identification of a constitutively active variant of LuxO that affects production of $\mathrm{HA}$ /protease and biofilm development in a non-O1, non-O139 Vibrio cholerae O110. Gene 369, 126-133. doi: 10.1016/j.gene.2005.10.031

Roig, F. J., Gonzalez-Candelas, F., and Amaro, C. (2011). Domain organization and evolution of multifunctional autoprocessing repeats-in-toxin (MARTX) toxin in Vibrio vulnificus. Appl. Environ. Microbiol. 77, 657-668. doi: 10.1128/AEM. 01806-10

Roland, F. P. (1970). Leg gangrene and endotoxin shock due to Vibrio parahaemolyticus: an infection acquired in New England coastal water. New Engl. J. Med. 282, 1306. doi: 10.1056/NEJM197006042822306

Satchell, K. J. F. (2007). MARTX, multifunctional autoprocessing repeats-in-toxin toxins. Infect. Immun. 75, 5079-5084. doi: 10.1128/IAI.00525-07

Schiavo, G., Poulain, B., Benfenati, F., DasGupta, B. R., and Montecucco, C. (1993). Novel targets and catalytic activities of bacterial protein toxins. Trends Microbiol. 1, 170-174. doi: 10.1016/0966-842X(93)90086-7

Shao, C. P., and Hor, L. (2000). Metalloprotease is not essential for Vibrio vulnificus virulence in mice. Infect. Immun. 68, 3569-3573. doi: 10.1128/IAI.68.6.35693573.2000

Sheahan, K. L., Cordero, C. L., and Satchell, K. J. F. (2007). Autoprocessing of the Vibrio cholerae RTX toxin by the cysteine protease domain. EMBO J. 26 2552-2561. doi: 10.1038/sj.emboj.7601700

Shen, A., Lupardus, P., Albrow, V. E., Guzetta, A., Powers, J. C., Garcia, K. C., et al. (2009). Mechanistic and structural insights into the proteolytic activation of Vibrio cholerae MARTX toxin. Nat. Chem. Biol. 5, 469-478. doi: 10.1038/nchembio. 178

Shinoda, S., and Miyoshi, S. (2006). "Hemolysins of Vibrio cholerae and other Vibrio species," in Comprehensive Sourcebook of Bacterial Protein Toxins, 3rd Ed, eds J. F. Alouf and M. R. Popoff (London: Academic Press), 748-762.

Shinoda, S., and Miyoshi, S. (2011). Proteases produced by vibrios. Biocontrol Sci. 16, 1-11. doi: 10.4265/bio.16.1

Silva, A. J., Leitch, G. J., Camilli. A., and Beitez, J. A. (2006). Contribution of hemagglutinin/protease and motility to the pathogenesis of el tor biotype cholera. Infect. Immun. 74, 2072-2079. doi: 10.1128/IAI.74.4.2072-2079. 2006

Stojiljkovic, I., and Perkins-Balding, D. (2002). Processing of heme and heme-containing proteins by bacteria. DNA Cell Biol. 21, 281-295. doi: $10.1089 / 104454902753759708$

Strom, M. S., and Paranjpye, R. N. (2000). Epidemiology and pathogenesis of Vibrio vulnificus. Microbes Infect. 2, 177-188. doi: 10.1016/S1286-4579(00) 00270-7

Sultan, Z., Miyoshi, S., and Shinoda, S. (2006). Presence of LuxS/AI-2 based quorum-sensing system in Vibrio mimicus: LuxO controls pretease activity. Microbiol. Immunol. 50, 407-417.

Sun, H. Y., Han, S. I., Choi, M. H., Kim, S. J., Kim, C. M., and Shin, S. H. (2006). Vibrio vulnificus metalloprotease $\mathrm{VvpE}$ has no direct effect on iron-uptake from human hemoglobin. J. Microbiol. 44, 537-547.

Takeuchi, H., Shibano, Y., Morihara, K., Fukushima, J., Inami, S., Keil, B., et al. (1992). Structural gene and complete amino acid sequence of Vibrio alginolyticus collagenase. Biochem. J. 281, 703-708.

Thompson, F. L., Hoste, G., Vandemeulebroecke, K., and Swing, J. (2003). Reclassification of Vibrio hollisae as Grimontia hollisae gen. nov., comb. nov. Int. J. Syst. Evol. Microbiol. 53, 1615-1617. doi: 10.1099/ijs.0. 02660-0
Tison, D. L., Nishibuchi, M., Greenwood, J. D., and Seidler, R. J. (1982). Vibrio vulnificus biotype 2: new biogroup pathogenic for eels. Appl. Environ. Microbiol. 44, 640-646.

Tong, Y., and Guo, M. (2009). Bacterial heme-transport proteins and their heme-coordination modes. Arch. Biochem. Biophys. 481, 1-15. doi: 10.1016/j.abb.2008.10.013

Trucksis, M., Galen, J. E., Michalski, J., Fasano, A., and Kaper, J. B. (1993). Accessory cholera enterotoxin (Ace), the third toxin of a Vibrio cholerae virulence cassette. Proc. Natl. Acad. Sci. U.S.A. 90, 5267-5271. doi: 10.1073/pnas.90.11.5267

Wang, H. X., Ayala, J. C., Benitez, J. A., and Silva, A. J. (2012). Interaction of the histone-like nucleotide structuring protein and the general stress response regulator RpoS at Vibrio cholerae promoters that regulate motility and hemagglutinin/protease expression. J. Bacteriol. 194, 1205-1215. doi: 10.1128/JB.05900-11

Wang, J., Sasaki, T., Maehara, Y., Nakao, H., Tsuchiya, T., and Miyoshi, S. (2008). Variation of extracellular proteases produced by Vibrio vulnificus clinical isolates: genetic diversity of the metalloprotease gene (vvp), and serine protease secretion by vvp-negative strains. Microbiol. Pathog. 44, 494-500. doi: 10.1016/j.micpath.2008.01.001

Wang, H. X., Wu, J. H., Ayala, J. C., Benitez, J. A., and Silva, A. J. (2011). Interplay among cyclic diguanylate, HapR, and the general stress response regulator (RpoS) in the regulation of Vibrio cholerae hemagglutinin/protease. J. Bacteriol. 193, 6529-6538. doi: 10.1128/JB.05166-11

Watanabe, H., Miyoshi, S., Kawase, T., Tomochika, K., and Shinoda, S. (2004). High growing ability of Vibrio vulnificus biotype 1 is essential for production of a toxic metalloprotease causing systemic diseases in humans. Microbiol. Pathog. 36, 117-123. doi: 10.1016/j.micpath.2003.10.001

Whitehead, N. A., Barnard, A. M., Slater, H., Simpson, N. J., and Salmond, G. P. (2001). Quorum-sensing in Gram-negative bacteria. FEMS Microbiol. Rev. 25, 365-404. doi: 10.1111/j.1574-6976.2001.tb00583.x

Yu, M. S., and Lee, C. Y. (1999). Expression and characterization of the prtV gene encoding a collagenase from Vibrio parahaemolyticus in Escherichia coli. Microbiology 145, 143-150. doi: 10.1099/13500872-145-1-143

Yun, J. H., Kim, H., Park, J. E., Lee, J. S., and Lee, W. (2012). Solution structure and dynamics of C-terminal regulatory domain of Vibrio vulnificus extracellular metalloprotease. Biochem. Biophys. Res. Commun. 430, 541-546.doi: 10.1016/j.bbrc.2012.11.117

Conflict of Interest Statement: The author declares that the research was conducted in the absence of any commercial or financial relationships that could be construed as a potential conflict of interest.

Received: 28 August 2013; paper pending published: 03 October 2013; accepted: 26 October 2013; published online: 18 November 2013.

Citation: Miyoshi S (2013) Extracellular proteolytic enzymes produced by human pathogenic Vibrio species. Front. Microbiol. 4:339. doi: 10.3389/fmicb.2013.00339 This article was submitted to Aquatic Microbiology, a section of the journal Frontiers in Microbiology.

Copyright (c) 2013 Miyoshi. This is an open-access article distributed under the terms of the Creative Commons Attribution License (CC BY). The use, distribution or reproduction in other forums is permitted, provided the original author(s) or licensor are credited and that the original publication in this journal is cited, in accordance with accepted academic practice. No use, distribution or reproduction is permitted which does not comply with these terms. 